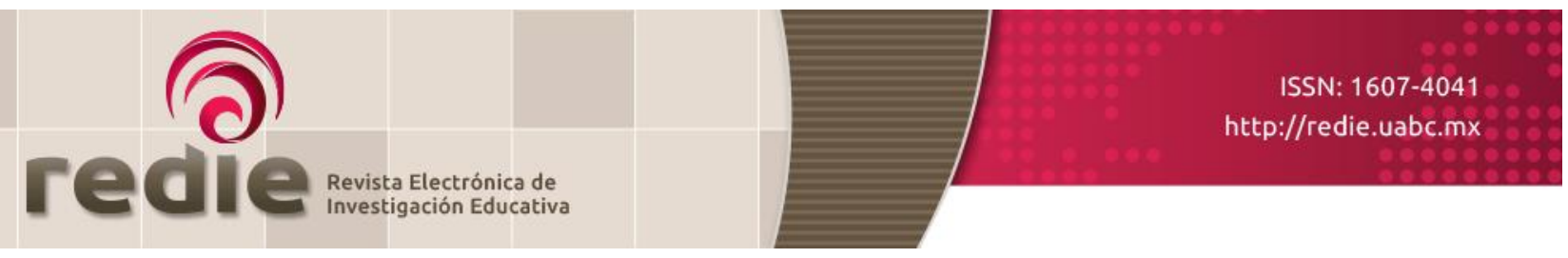

Vol. 20, Núm. 2, 2018

\title{
Funcionamiento democrático y ABP: factores determinantes para la inclusión y el rendimiento del alumnado
}

\section{Democratic Functioning and PBL: Determining Factors for Inclusion and Academic Achievement}

Andoni Arguiñano Madrazo (*) inodnaa@gmail.com

Iñaki Karrera Juarros (*) inodnaa@gmail.com

Maite Arandia Loroño (*) maite.arandia@ehu.eus

(*) Universidad del País Vasco

(Recibido: 16 de junio de 2016; Aceptado para su publicación: 8 de diciembre de 2016)

Cómo citar: Arguiñano, A., Karrera, I. y Arandia, M. (2018). Funcionamiento democrático y ABP: factores determinantes para la inclusión y el rendimiento del alumnado. Revista Electrónica de Investigación Educativa, 20(2), 103-112.

https://doi.org/10.24320/redie.2018.20.2.1450

\section{Resumen}

Este trabajo analiza cómo el funcionamiento democrático y la metodología basada en proyectos (ABP) influyen en el rendimiento del alumnado. Se relacionan las evidencias descritas en la literatura científica sobre la mejora del rendimiento y la inclusión con los resultados logrados por el alumnado de Antzuola en las pruebas de diagnóstico realizadas por el Instituto Vasco de Investigación Educativa. La perspectiva metodológica desde la cual se afronta el estudio es cuantitativa; se comparan los resultados de los años 2010, 2011 y 2013 del alumnado de Antzuola con el alumnado de la Comunidad Autónoma Vasca -que realizaron las mismas pruebas en diferentes centros y estratos lingüísticos. El estudio concluye que las prácticas educativas desarrolladas en el centro generan mejores resultados académicos, mostrando un porcentaje significativamente menor de alumnos con resultados por debajo de la media, lo que puede ser entendido como un mayor nivel de inclusión.

Palabras clave: Funcionamiento democrático, inclusión, Aprendizaje Basado en Proyectos (ABP), rendimiento académico, pruebas estandarizadas.

\begin{abstract}
This work analyzes how democratic functioning and project-based learning methodology (PBL) influence student performance. A link is established between, on the one hand, findings described in scientific literature on performance improvement and inclusion, and on the other, results obtained by Antzuola students in diagnostic testing carried out by the Basque Institute for Educational Research. The methodological perspective of the study is quantitative; it compares results obtained by Antzuola students and those of students from the Basque Autonomous Community who took the same tests in different centers and linguistic strata in 2010, 2011, and 2013. The study concludes that educational
\end{abstract}


practices employed in the center produce better academic results, with a significantly lower percentage of students with below-average results. This can be interpreted as a higher level of inclusion.

Keywords: Democratic management, Inclusion, Project Based Learning (PBL), academic achievement, standardized tests.

\section{Introducción}

El incremento de estudiantes universitarios en el municipio de Antzuola entre 1996 y 2011 es significativo. Ha pasado de 5.82 a 22.69\%, mientras que en los pueblos de la comarca el incremento ha sido de un 10\% (Instituto Vasco de Estadística [EUSTAT], 2011). Una de las posibles causas de la mejora de resultados académicos o del interés creciente por estos puede ser el centro educativo, ya que este es uno de los factores clave que incide en el valor que la comunidad concede a la educación.

Analizar el centro educativo del pueblo, su sistema y los resultados que obtiene puede ayudar a explicar en cierta medida dicho incremento de estudiantes universitarios. "Antzuola Herri Eskola" es un centro de Educación Infantil y Primaria que durante 30 años se ha esforzado por generar un nuevo marco metodológico partiendo de las necesidades de su comunidad. La principal y más extensa literatura científica que describe el funcionamiento del centro es aquella en la que se relatan experiencias vividas por el equipo docente, firmadas como Antzuola Herri Eskola (1999; 2000; 2001a; 2001b; 2003; 2004a; 2004b; 2006; 2008; 2014). Los artículos, describen un centro que abandona el modelo de transmisión de conocimiento tradicional definido por Freire (1994) como "modelo de educación bancaria" por otro que fomenta la idea del estudiante como investigador que se pregunta, formula hipótesis y experimenta. Un centro que aborda el aprendizaje mediante el uso de una metodología que prioriza el crecimiento personal y colectivo para garantizar el bienestar y la autoestima, siempre con la intención de "empoderar" al alumnado. Además, el centro ha expresado en reiteradas ocasiones que este empoderamiento se extiende a otros agentes de la comunidad (madres, padres y administración) utilizando el diálogo. Una idea de empoderamiento que responde a la definición dada por Torres (2009), para quien el empoderamiento en la educación es un proceso que tiene como objetivo el cambio individual y social, que ayuda a adquirir conciencia sobre las capacidades y potencialidades mediante la reflexión, la crítica, la concienciación, la acción y una estrecha relación con el entorno. Esta metodología genera en el alumnado el bienestar que el centro pretende.

Parte de la sociedad entiende esta metodología como una manera de proceder poco eficiente al no someter al alumnado a pruebas estandarizadas de evaluación que en el futuro cercano, a lo largo de su vida académica, deberá superar. Sin embargo, los estudios previos que describen el funcionamiento de Antzuola descubren varias actividades y políticas descritas en la literatura científica como actividades eficientes que generan en los centros una posibilidad de cambio dirigida a la mejora de resultados y a un mayor nivel de inclusión de los mismos.

Según Johari y Bradshaw (2008) el Aprendizaje Basado en Proyectos (ABP), convierte el aprendizaje del alumnado en un quehacer agradable. Según Blumenfeld et al. (1991) esta metodología consigue que mejore el producto final elaborado por los alumnos debido a lo significativo de la misma. Thomas (2000) afirma que consigue la implicación del alumnado en la toma de decisiones, algo que genera una construcción de conocimiento colectivo, además de capacitar a los estudiantes para autoevaluar su trabajo y generar en ellos un sentimiento de responsabilidad hacia el aprendizaje. El estudio realizado por Liu, Wang, Tan, Koh y Ee (2009) muestra cómo la percepción sobre el aprendizaje que tienen los estudiantes es más positiva que utilizando otras metodologías, algo que concuerda con el realizado por Willard y Duffrin (2003), quienes descubrieron que la ABP produce mayores índices de satisfacción entre los estudiantes que el sistema tradicional de enseñanza. El carácter comunicativo de la ABP consigue que el alumnado aprenda a presentar y exponer temáticas diversas. Mioduser y Betzer (2008) añaden a las cualidades descritas la capacidad que tiene para activar conocimientos previos en los alumnos, para mejorar su razonamiento crítico y la metacognición, así como para desarrollar las destrezas de estudio autónomo y la capacidad de solucionar problemas de manera autónoma. 
Antzuola Herri Eskola se caracteriza por su metodología basada en proyectos y por el empoderamiento del alumnado y de los agentes sociales que parecen incidir en los aprendizajes del alumnado. En este sentido, hay evidencias científicas recientes que destacan el papel que tiene la participación educativa y sus implicaciones en la superación de las desigualdades (Moliner, Traver, Ruiz y Segarra, 2016), así como en la mejora académica del alumnado (Bemak y Cornely, 2002; Santa Cruz, Siles y Vrecer, 2011). Dar voz a las niños, tal y como hace Antzuola Herri Eskola, se describe en la literatura científica como una premisa que favorece el aprendizaje.

Una de las razones de la importancia de la participación del alumnado, según Sánchez (1999), es la idea de que cuando se da importancia a los intereses de los propios participantes se incrementa la motivación por el aprendizaje y sus conocimientos. Todos los procesos descritos hasta ahora son posibles gracias a un equipo directivo que trabaja en estrecha colaboración con todos los agentes de la comunidad aprovechando las diferentes capacidades para lograr un beneficio común.

El liderazgo escolar es absolutamente crucial. De hecho, el análisis que realiza la organización Best Evidence Synthesis (BES) de los resultados de la investigación mundial sobre el papel del liderazgo relacionado con los resultados del aprendizaje estudiantil, concluyó que un factor dos veces más poderoso que cualquier otra variable en afectar al aprendizaje del alumno era el grado en que "el director de la escuela participa como estudiante" junto con los maestros en las mejoras escolares Robinson, 2009 (como se cita en Fullan, 2011, p. 32). El profesorado asume funciones de liderazgo en sus respectivas áreas y ámbitos (Elmore, 2000). El liderazgo comienza a verse cada vez menos como una tarea única que recae en un individuo y más como una responsabilidad de toda la comunidad, que es asumida por distintas personas según sus competencias y momentos. En esta nueva visión la principal tarea del director es desarrollar la capacidad de liderazgo de los demás, estimulando el talento y la motivación. Un liderazgo que ha demostrado conseguir escuelas más equitativas, más participativas, más inclusivas y, en definitiva, escuelas de mayor calidad.

Tener en cuenta a la comunidad, ponderar a los estudiantes dándoles voz en las decisiones, tener fe en las personas y en su capacidad para resolver problemas de manera democrática o preocuparse por el bienestar de todos (por encima de todo) acerca el centro a la filosofía de las escuelas democráticas descritas por Apple y Beane (1997), una filosofía de centro que, como señalan Racionero y Padrós (2010), resulta ser una acción educativa exitosa que transforma el contexto socio-cultural y mejora el aprendizaje. Apple y Beane (1997) hacen una aproximación interesante a la democracia y democratización de los centros. Entienden que el rasgo central de la democracia es el bien común. Рara avanzar en ese proceso de democratización escolar concretan las preocupaciones centrales que ha de tener una escuela democrática:

- La preocupación por la dignidad y los derechos de los individuos y las minorías.

- Una comprensión de que la democracia no es tanto un "ideal" que se debe perseguir como un conjunto "idealizado" de valores que debemos vivir y que deben guiar nuestra vida como pueblo.

- La organización de instituciones sociales para promover y ampliar la forma de vida democrática y el centro educativo como herramienta impulsora del cambio.

Un centro educativo que cumpliera con las premisas descritas a lo largo de esta primera parte del marco teórico debería de obtener mejores resultados académicos y en cuanto a inclusión se refiere, si entendemos que una educación inclusiva hace referencia al derecho a la educación que ha de ser garantizado a todas las personas por imperativos éticos, de justicia y equidad. Esta inclusión debería de albergar a todos y cada uno de los estudiantes dentro del marco de las competencias básicas o claves concertadas en el seno de la Unión Europea.

En otro sentido, Bolívar y López (2009, p. 53) entienden la exclusión educativa como "los casos en los que hay una dificultad de acceso, mantenimiento o abandono en el sistema educativo reglado". Por lo que el número de estudiantes que repite curso en un centro puede ser un predictivo de inclusión o de riesgo de 
exclusión. Por ello, analizar el nivel de rendimiento de un centro puede ser una manera fiable de medir el nivel de exclusión o inclusión escolar que se da en el mismo (López de la Nieta, 2008). La idea de utilizar el rendimiento escolar como reflejo del fracaso-éxito escolar ha sido explorado antes por Loreman (2014), y previamente por Dyson, Farrel, Polat, Hutcheson y Gallanaugh (2004).

El objetivo del estudio realizado es determinar en qué medida responde el centro a las necesidades de su alumnado sin importar los impedimentos que éste tenga. Los tamaños de los grupos son de similar ratio que el de los distintos centros de la Comunidad Autónoma Vasca (en adelante CAV). Además, el índice socioeconómico del centro también es inferior que el de la mayoría de los centros con los que se hacen las comparaciones y el número de inmigrantes en el centro duplica el porcentaje de la media de la cav. Aún así, los puntos antes mencionados -como incorporar en el centro las voces de todos los agentes de la comunidad para reducir las brechas culturales que podrían encontrarse fuera del aula, o el trabajar desde el interés de los estudiantes y hacerlos partícipes de las decisiones del centro y de su currículum, además de otras medidas que complementan éstas-, no hacen más que acrecentar la capacidad inclusiva del centro y, como consecuencia, reducen el riesgo de exclusión escolar.

Los objetivos de la investigación fueron: 1) Determinar si la metodología aplicada en Antzuola Herri Eskola es capaz de responder de manera satisfactoria a las pruebas estandarizadas de diagnóstico que realiza el ISEI-IVEI, y 2) Comprobar si el nivel de inclusión que obtiene el centro de Antzuola Herri Eskola es mayor, igual o menor que el resto de centros o estratos analizados.

\section{Método}

La metodología utilizada en este estudio fue de carácter cuantitativo longitudinal, permitiendo analizar en profundidad el objeto de estudio al observar los resultados que el centro ha obtenido en las pruebas de diagnosis a lo largo de diferentes años, minimizando así el sesgo de la observación casual al analizar sólo los resultados de un año.

Los participantes del análisis estadístico fueron todos los estudiantes de los centros públicos de la Comunidad Autónoma Vasca (CAV) que realizaron las pruebas de diagnóstico en los años 2010, 2011 y 2013 y la media del alumnado de la CAV de centros tanto públicos como concertados. Un total de 81,440 alumnos. Los datos que se recogen a lo largo de este estudio, exceptuando aquellos ligados a Antzuola y el centro de secundaria, se recopilaron de los informes ejecutivos que el Instituto Vasco de Educación e Investigación Educativa (ISEI-IVEI) tiene visibles en su página web. ${ }^{1}$ El número de alumnos que corresponde a cada estrato o centro son los siguientes: 1) Antzuola en Bruto, 38 alumnos con un ISEC (Economic, Social and Cultural Status) medio. La estadística de Antzuola en Bruto no discrimina los resultados de ningún alumno, manteniendo los resultados de alumnos que en el análisis realizado por ISEC son descartados por problemas en la realización de las pruebas o por ser alumnos de incorporación tardía al sistema de educación. 2) Centro ESO, 260 alumnos con un ISEC medio; 3) Pública "A", 779 alumnos con un ISEC bajo; 4) Pública "B", 2,885 alumnos con un ISEC medio; 5) Pública "D" 20,168 alumnos con un ISEC medio-alto y 6) la Media de la CAV, 55,541 alumnos.

Para abordar el análisis del rendimiento académico se han recogido los resultados del alumnado en las pruebas de Evaluación de Diagnóstico realizadas por el instituto ISEI-IVEI para su posterior comparación con distintos estratos de la enseñanza pública, así como de las medias de la caV recurriendo al análisis estadístico mediante el programa SPSS v22.

El instituto ISEI-IVEI es un servicio de apoyo a la educación, dependiente de la Viceconsejería de Educación (Gobierno Vasco, 2001), encargada de diseñar y desarrollar proyectos de evaluación del sistema educativo de la CAV. Las pruebas de Evaluación de Diagnóstico valoran el nivel de desarrollo de las competencias básicas de cada uno de los alumnos escolarizados de 20. grado de la Educación Secundaria Obligatoria (ESO), de ahí que se haya decidido utilizar los resultados de las pruebas de Evaluación de Diagnóstico como medidores del nivel de riesgo de exclusión en el que se encuentran los estudiantes. Este

\footnotetext{
${ }^{1}$ http://www.isei-ivei.hezkuntza.net/web/guest
} 
instrumento (validado por un órgano de la CAv) posibilita la comparación de resultados a nivel de la Comunidad Autónoma.

Los resultados de ISEI-IVEI recopilados organizaron tal como se hace en el informe ejecutivo. De este modo, clasifican las medias de los resultados por cada alumno y centro educativo en tres grupos: 1) Por debajo de la media, menor a 200 puntos; 2) en la media, entre 201 y 300 puntos; y 3) sobre la media, superior a 301 puntos.

Mediante la observación de resultados, según las diferentes competencias evaluadas por ISEI, se cruzaron los datos. Para la obtención de los resultados que se muestran a continuación se utilizó el análisis de varianza (ANOVA de un factor), con el test de Bonferroni para comparaciones múltiples a un nivel de significación $p<0.05$, que permite contrastar los resultados de los tipos de centros escolares a través de combinaciones por pares de centros para observar si existen diferencias entre los resultados de los centros y estratos analizados, y si estas diferencias son significativas.

\section{Resultados}

Tras procesarlos los datos en el SPSS v22, los resultados obtenidos son los que aparecen en la tabla I.

Tabla I. Resumen de procesamiento de datos

\begin{tabular}{c|c|c|c|c|c|c}
\hline \multicolumn{2}{c}{ Casos } \\
\hline \multirow{2}{*}{ Casos } & Válidos & Porcentaje & $\mathrm{N}$ & Porcentaje & $\mathrm{N}$ & Porcentaje \\
\cline { 2 - 7 } & 317,497 & 97.4 & 8,332 & 2.6 & 325,829 & 100 \\
\hline
\end{tabular}

Como se observa en la tabla II, los datos de Antzuola tienen un menor porcentaje de alumnos bajo la media, este porcentaje correlaciona con los 3 centros con mejores resultados: Centro Eso, Estrato D y Media CAV. Los centros con el porcentaje más alto con alumnos por encima de la media son Antzuola y Media CAV, con un $24.3 \%$.

Tabla II. Resultados por tipo de centro

\begin{tabular}{l|c|c|c|c|c|c}
\hline & $\begin{array}{c}\text { Antzuola } \\
\text { en Bruto (b) }\end{array}$ & $\begin{array}{c}\text { Centro Eso } \\
\text { (c) }\end{array}$ & $\begin{array}{c}\text { Estrato A } \\
\text { (d) }\end{array}$ & $\begin{array}{c}\text { Estrato B } \\
(\mathrm{e})\end{array}$ & $\begin{array}{c}\text { Estrato D } \\
(\mathrm{f})\end{array}$ & $\begin{array}{c}\text { Media CAV } \\
(\mathrm{g})\end{array}$ \\
\hline Bajo Media & $17.9 \%$ & $20.1 \%$ & $53.6 \%$ & $34 \%$ & $19.8 \%$ & $20.1 \%$ \\
& $\mathrm{~b}$ & $\mathrm{~b}$ & $\mathrm{C}$ & $\mathrm{d}$ & $\mathrm{b}, \mathrm{e}$ & $\mathrm{b}, \mathrm{f}$ \\
En la Media & $57.9 \%$ & $58.7 \%$ & $34.6 \%$ & $52.9 \%$ & $56.4 \%$ & $55.5 \%$ \\
Sobre la & $\mathrm{d}, \mathrm{e}, \mathrm{f}$ & $\mathrm{b}, \mathrm{f}$ & $\mathrm{C}$ & $\mathrm{d}$ & $\mathrm{b}, \mathrm{e}$ & $\mathrm{f}$ \\
media & $24.3 \%$ & $21.2 \%$ & $11.8 \%$ & $13.1 \%$ & $23.8 \%$ & $24.3 \%$ \\
\hline
\end{tabular}




\subsection{Resultados por asignatura}

Tabla III. Resultados en la competencia de Euskara

\begin{tabular}{l|c|c|c|c|c|c|c|c}
\hline & $\begin{array}{c}\text { Antzuola } \\
\text { en Bruto }\end{array}$ & $\begin{array}{c}\text { Centro } \\
\text { ESO }\end{array}$ & $\begin{array}{c}\text { Estrato } \\
\text { A }\end{array}$ & $\begin{array}{c}\text { Estrato } \\
\text { B }\end{array}$ & $\begin{array}{c}\text { Estrato } \\
\text { D }\end{array}$ & $\begin{array}{c}\text { Media } \\
\text { CAV }\end{array}$ & P & V \\
\hline Bajo & $25.7 \%$ & $21 \%$ & $95 \%$ & $56 \%$ & $26 \%$ & $33.8 \%$ & .000 & .182 \\
Media & b & b & c & d & b & & & \\
Sobre la & $31.4 \%$ & $26 \%$ & $0.7 \%$ & $9.4 \%$ & $25 \%$ & $23.8 \%$ & & \\
media & b & b & c & d & b & & & \\
\hline
\end{tabular}

Tabla IV. Resultados en la competencia de Castellano

\begin{tabular}{l|c|c|c|c|c|c|c|c}
\hline & Antzuola & Centro & Estrato & Estrato & Estrato & Media & P & V \\
& en Bruto & ESO & A & B & D & CAV & & \\
\hline Bajo Media & $17.1 \%$ & $23.2 \%$ & $44 \%$ & $20 \%$ & $14 \%$ & $12.2 \%$ & .000 & .116 \\
Sobre la & b,c & $\mathrm{C}$ & $\mathrm{d}$ & $\mathrm{C}$ & $\mathrm{b}$ & $\mathrm{a}$ & & \\
media & $14.3 \%$ & $21 \%$ & $12.7 \%$ & $21.7 \%$ & $25.9 \%$ & $24 \%$ & & \\
\hline
\end{tabular}

El porcentaje menor de alumnos bajo la media lo tiene la Media de la CAV, los resultados de este centro no tienen correlación significativa con ninguno de los demás centros o estratos analizados. Antzuola, por lo tanto, se encuentra en el segundo escalón junto al Centro ESO y el Estrato D. En el porcentaje de encima de la media sólo se encuentra por encima del Estrato A, pero no existen diferencias significativas entre Antzuola y el resto de centros o estratos analizados.

Tabla V. Resultados en la competencia de Matemática

\begin{tabular}{|c|c|c|c|c|c|c|c|c|}
\hline & $\begin{array}{l}\text { Antzuola } \\
\text { en Bruto }\end{array}$ & $\begin{array}{c}\text { Centro } \\
\text { ESO }\end{array}$ & $\begin{array}{c}\text { Estrato } \\
\text { A }\end{array}$ & $\begin{array}{c}\text { Estrato } \\
\text { B }\end{array}$ & $\begin{array}{c}\text { Estrato } \\
\text { D }\end{array}$ & $\begin{array}{l}\text { Media } \\
\text { CAV }\end{array}$ & $P$ & V \\
\hline Bajo Media & $\begin{array}{c}17.1 \% \\
b, c\end{array}$ & $\begin{array}{c}16 \% \\
b\end{array}$ & $\begin{array}{c}41.8 \% \\
C\end{array}$ & $\begin{array}{c}26.8 \% \\
b\end{array}$ & $15.6 \%$ & $15.7 \%$ & .000 & .100 \\
\hline $\begin{array}{l}\text { Sobre la } \\
\text { media }\end{array}$ & $\begin{array}{c}25.7 \% \\
b, c\end{array}$ & $\begin{array}{c}19.1 \% \\
b, c\end{array}$ & $\begin{array}{c}15.7 \% \\
C\end{array}$ & $\begin{array}{c}14.9 \% \\
\mathrm{C}\end{array}$ & $\begin{array}{c}22.3 \% \\
b\end{array}$ & $\begin{array}{c}26 \% \\
b\end{array}$ & & \\
\hline
\end{tabular}

Los resultados de alumnos bajo la media de Antzuola se correlacionan significativamente con los obtenidos por el Centro ESO y el Estrato B. Siendo mejores resultados únicamente los del estrato D y la Media de la CAV. En cuanto al porcentaje de alumnos por encima de la media, los resultados de Antzuola son los mejores junto a los del Estrato D, la Media de la CAV y el Centro Eso.

Tabla VI. Resultados en la competencia de Inglés

\begin{tabular}{l|c|c|c|c|c|c|c|c}
\hline & $\begin{array}{c}\text { Antzuola } \\
\text { en Bruto }\end{array}$ & $\begin{array}{c}\text { Centro } \\
\text { ESO }\end{array}$ & $\begin{array}{c}\text { Estrato } \\
\mathrm{A}\end{array}$ & $\begin{array}{c}\text { Estrato } \\
\mathrm{B}\end{array}$ & $\begin{array}{c}\text { Estrato } \\
\mathrm{D}\end{array}$ & $\begin{array}{c}\text { Media } \\
\text { CAV }\end{array}$ & $\mathrm{P}$ & $\mathrm{V}$ \\
\hline Bajo Media & $\begin{array}{c}6.7 \% \\
\mathrm{~b}\end{array}$ & $\begin{array}{c}22.1 \% \\
\mathrm{~b}\end{array}$ & $\begin{array}{c}24.1 \% \\
\mathrm{~b}\end{array}$ & $40.4 \%$ & & $16.9 \%$ & .000 & .042 \\
Sobre la & $\begin{array}{c}40 \% \\
\mathrm{~b}\end{array}$ & $\begin{array}{c}14.7 \% \\
\mathrm{~b}\end{array}$ & $\begin{array}{c}16 \% \\
\mathrm{~b}\end{array}$ & $6.2 \%$ & & $\begin{array}{c}24.6 \% \\
\mathrm{~b}\end{array}$ & & \\
media & $\mathrm{b}$ & & & & \\
\hline
\end{tabular}

El centro con el menor porcentaje de alumnos bajo la media y el más alto en alumnos sobre la media es el de Antzuola, pero debido a que esta asignatura sólo se ha evaluado en uno de los tres años el programa no encuentra diferencias significativas con los resultados de centros y estratos como el centro ESo, el Estrato A o la Media de la CAV. 


\section{Discusión}

Todos los presupuestos educativos dirigidos al trabajo en comunidad por y para los estudiantes que desencadenaron en una posibilidad de mejora del rendimiento e inclusión dejaron de ser meras especulaciones para convertirse en mejoras reales y palpables. El centro de Antzuola Herri Eskola ha demostrado que por decisión comunitaria, sin realizar en sus aulas pruebas de evaluación de carácter sumativo, con un índice socioeconómico medio (medio-bajo dependiendo del año) y un número de estudiantes de incorporación tardía al sistema educativo por encima de la Media de la CAV, en los años 2010 y 2013 consiguió resultados satisfactorios.

Antzuola en Bruto, con un 17.9\%, es el centro que menor número de estudiantes deja rezagados frente al $20.1 \%$ del Centro ESO o el 53\% del estrato A, del 34\% del estrato B, del $19.8 \%$ del estrato D o del $20.1 \%$ de la Media de la CAV. Por tanto, el nivel de inclusión de Antzuola está por encima del resto aunque la diferencia no sea significativa respecto al Centro ESO, el estrato D o la Media de la CAV. La distancia de la significación en los resultados no es muy amplia, ya que la $v$ de Cramer muestra que las distancias oscilan entre 0.042 y 0.182. La cercanía de la distancia, vista la diferencia en los porcentajes, podría deberse a que se están comparando porcentajes de 38 estudiantes con porcentajes como el de la CAV, que consta de más 50,000 estudiantes. Aunque no pueden menospreciarse los resultados, ya que esos 38 alumnos conforman el universo de Antzuola Herri Eskola.

Como ya se expresó en el marco teórico, este colectivo de estudiantes que no supera la nota media es el colectivo que más posibilidades tiene de ser excluido del sistema educativo. Según Bolívar y López (2009), a mayor porcentaje de suspensos, lo que en este caso puede entenderse como alumnado por debajo de la media, mayor porcentaje de posibles repetidores. Como expresan López de la Nieta (2008) y Loreman, (2013) estos repetidores son los que abandonan prematuramente la educación, por lo que son el grupo con mayor riesgo de exclusión. Antzuola Herri Eskola es el centro que ha demostrado que tiene menos porcentaje de estudiantes en riesgo de exclusión, lejos de los centros o estratos analizados así como de la Media de la CAV, por lo que puede afirmarse que el sistema pedagógico de Antzuola es, entre los centros estudiados, el que mejores resultados da en cuanto al nivel de inclusión. Más aún, si atendemos a las variables "alumnado de incorporación tardía" e "índice socioeconómico" -pues es el centro con mayor número de alumnos pertenecientes a esta primera categoría y el segundo de los analizados con el índice socioeconómico más bajo igualado por el Centro ESO- ha conseguido que sus índices de fracaso sean significativamente menores a los del resto de centros educativos y estratos analizados. En cuanto al alto rendimiento las lecturas son similares. Antzuola en Bruto es el centro con mayor porcentaje de alumnos sobre la media junto con la Media de la CAV.

\section{Conclusiones}

En cuanto a la posibilidad de que el funcionamiento democrático sea el factor determinante de los resultados del centro y, por consiguiente, del nivel de inclusión que éste consigue, es algo que no puede afirmarse debido a que existen otros factores a estudiar que podrían tener el mismo impacto en los resultados. Sin embargo, sí puede defenderse la idea de que este funcionamiento democrático ha sido un factor que ha influido y posibilitado el resto de variables que podrían incidir tanto o más en el nivel de inclusión o en el nivel de rendimiento. De ahí que sin un funcionamiento democrático la voz del profesorado difícilmente se tomaría en cuenta. Del mismo modo, la metodología por proyectos que se implantó y se ha desarrollado hasta la fecha, nació a raíz del consenso entre padres, profesores y administración.

Aunque todas estas cuestiones tienen varias características que influyen en el rendimiento académico, no ha podido determinarse si ha mejorado o cuánto ha mejorado el mismo. Para ello se necesitaría un histórico de resultados que no existe en la actualidad debido a que no hay pruebas similares a las realizadas por ISEI-IVEI en tiempos anteriores. Sin embargo, puede intuirse dicha mejora observando el incremento del porcentaje de universitarios que ha experimentado el municipio de Antzuola. Рara documentar esta mejora queda la opción de entrevistar a diversas generaciones y analizar los resultados. De este modo se podría examinar si realmente se encuentra una mejora de resultados. Por otro lado, 
tampoco puede afirmarse que el incremento de estudiantes universitarios en el pueblo se deba a las políticas democráticas que se dan en el centro, pero sí puede afirmarse que estas políticas dieron pie a las medidas tomadas el centro en cuanto a la pedagogía que impulsa, y éstas, a su vez, han conseguido mejorar el nivel de rendimiento del alumnado. Se puede decir que estas políticas han abierto el camino para inculcar en el sentir del pueblo la importancia de los estudios, así como la posibilidad de acceder a ellos y no abandonarlos.

Los resultados que el centro ha obtenido son buenos. En principio se manejó la hipótesis de que los resultados de Euskara podrían deberse a las características de la región y del pueblo en particular, pero en lo concerniente al resto de competencias genéricas las significativas diferencias existentes entre Antzuola y el resto de centros educativos demostraron que son correctas las investigaciones que afirman que las políticas de centro, el trabajo por proyectos y la influencia de la comunidad mejoran los resultados de los estudiantes.

La investigación partió del supuesto de que en el resto de los centros y estratos se imparte una didáctica convencional, obviando que existen centros agrupados en redes de innovación educativa que podrían compararse entre sí y analizar el rendimiento académico y nivel de inclusión que cada propuesta educativa obtiene.

Siendo conscientes de que existen variables no medidas, puede afirmarse que las acciones educativas dirigidas a la búsqueda de la igualdad y la equidad logradas gracias a los procesos democráticos impulsados han dado resultados en Antzuola Herri Eskola, al conseguir que este centro tenga resultados satisfactorios.

\section{Referencias}

Antzuola Herri Eskola. (1999). Infantil y primaria, ¿dos mundos independientes? Cuadernos De Pedagogía, $282,40-44$.

Antzuola Herri Eskola. (2000). Gestión del currículum y concepciones del alumnado. Cuadernos de Pedagogía, 290, 70-73.

Antzuola Herri Eskola. (2001a). Niños y niñas investigadoras: ¿de qué hablamos? Aula de Infantil, 1, 7-14.

Antzuola Herri Eskola. (2001b). El qué, cuándo, para qué... de las matemáticas. Aula de Innovación Educativa, 10(103-104), 20-24.

Antzuola Herri Eskola. (2003). La cantidad y... i cantidad de significados! Aula de Infantil, 13, 6-8.

Antzuola Herri Eskola. (2004a). Una comunidad que acaba. Aula de Innovación Educativa, 140(132), 40-46.

Antzuola Herri Eskola. (2004b). En la escuela, lo que digo yo también es importante. Aula de Innovación Educativa, 132, 41-46.

Antzuola Herri Eskola. (2006). El viaje a marte. Cuadernos de Pedagogía, 355, 55-58.

Antzuola Herri Eskola. (2008). Experiències primària: Un claustre que indaga i aprèn com a comunitat. L’escola d`Antzuola [Experiencias en primaria: un claustro que indaga y aprende como comunidad]. Perspectiva Escolar, 323, 27-31. 
Antzuola Herri Eskola. (2014). La escuela de Antzuola: Las personas y sus interacciones en contextos de aprendizaje inclusivo. Características y Experiencias. XXI Encuentro de Consejos Escolares Autonómicos y del Estado. Recuperado de

http://www.mecd.gob.es/dctm/cee/encuentros/xxiiencuentro/22encuentroceayedocumentofinaljpa.pdf? documentld=0901e72b8194fe50

Apple, M. W. y Beane, J. A. (1997). Escuelas democráticas (1a. ed.). Madrid: Morata.

Bemak, F. y Cornely, L. (2002). The SAFI model as a critical link between marginalized families and schools: A literature review and strategies for school counselors. Journal of Counseling \& Development, 80(3), 322331.

Blumenfeld, P. C., Soloway, E., Marx, R. W., Krajcik, J. S., Guzdial, M. y Palincsar, A. (1991). Motivating project-based learning: Sustaining the doing, supporting the learning. Educational Psychologist, 26(3-4), 369-398.

Bolívar, A. y López, L. (2009). Las grandes cifras del fracaso y los riesgos de exclusión educativa. Profesorado: Revista de Currículum y Formación del Profesorado, 13(3), 51-78.

Dyson, A., Farrell, P., Polat, F., Hutcheson, G. y Gallannaugh, F. (2004). Inclusion and pupil achievement. London: Department for Education and Skills.

Elmore, R. F. (2000). Building a new structure for school leadership. Washington, DC: Albert Shanker Institute.

Freire, P. (1994). Pedagogia do oprimido [Pedagogía del oprimindo]. Río de Janeiro: Paz e Terra.

Fullan, M. (2011). Investigación sobre el cambio educativo: Presente y futuro. Revista Digital de Investigación Lasaliana, 3, 31-35.

Instituto Vasco de Evaluación e Investigación Educativa (2011). El funcionamiento y organización del Instituto Vasco de Evaluación e Investigación Educativa No Universitaria (IVEI). Decreto 21/2001U.S.C. publicado en el Boletín Oficial del País Vasco (2001, 5 de abril).

Johari, A. y Bradshaw, A. C. (2008). Project-based learning in an internship program: A qualitative study of related roles and their motivational attributes. Educational Technology Research and Development, 56(3), 329-359.

Liu, W. C., Wang, C. J., Tan, O. S., Koh, C. y Ee, J. (2009). A self-determination approach to understanding students' motivation in project work. Learning and Individual Differences, 19(1), 139-145.

López de la Nieta, M. (2008). Sistema educativo y desigualdad: Un estudio sobre los logros educativos de la población y algunos aspectos del fracaso escolar. Documento presentado en Políticas y Bienes Sociales: Procesos de Vulnerabilidad y Exclusión Social (pp. 321-408), Oviedo, España.

Loreman, T. (2014). Measuring inclusive education outcomes in Alberta, Canada. International Journal of Inclusive Education, 18(5), 459-483.

Mioduser, D. y Betzer, N. (2008). The contribution of project-based-learning to high-achievers' acquisition of technological knowledge and skills. International Journal of Technology and Design Education, 18(1), 5977.

Moliner, O., Traver, J. A., Ruiz, M. A. y Segarra, T. (2016). Estrategias que inciden en los procesos de democratización de la escuela. Una aproximación teórica. Revista Electrónica de Investigación Educativa, 18(2), 116-129. Recuperado de https://redie.uabc.mx/redie/article/view/1110 
Racionero, S. y Padrós, M. (2010). The dialogic turn in educational psychology. Journal of Psychodidactics, 15(2), 143-162.

Sánchez, M. (1999). Voices inside schools. La Verneda-Sant Martí: a school where people dare to dream. Harvard Educational Review, 69(3), 320-336.

Santa Cruz, I., Siles, G. y Vrecer, N. (2011). Invest for the long term or attend to immediate needs? schools and the employment of less educated youths and adults. European Journal of Education, 46(2), 197-208.

Thomas, J. W. (2000). A review of research on project-based learning. Recuperado de http://www.bobpearlman.org/BestPractices/PBL Research.pdf

Torres, A. (2009). La educación para el empoderamiento y sus desafíos. Sapiens: Revista Universitaria de Investigación, 10(1), 89-108.

Willard, A. K. y Duffrin, M. W. (2003). Utilizing project-based learning and competition to develop student skills and interest in producing quality food items. Journal of Food Science Education, 2(4), 69-73. 